\title{
Existence results for Gradient elliptic systems with nonlinear boundary conditions
}

\author{
Julián Fernández BONDER, Sandra MARTÍNEZ \& Julio D. ROSSI \\ Departamento de Matemática, FCEyN \\ UBA (1428) Buenos Aires \\ Argentina \\ e-mail: jfbonder@dm.uba.ar \\ smartin@dm.uba.ar \\ jrossi@dm.uba.ar \\ http://mate.dm.uba.ar/ jfbonder, \\ http://mate.dm.uba.ar/ jrossi
}

Abstract. We prove the existence of nontrivial solutions to the system

$$
\Delta_{p} u=|u|^{p-2} u, \quad \Delta_{q} v=|v|^{q-2} v
$$

on a bounded set of $\mathbb{R}^{N}$, with nonlinear coupling at the boundary given by

$$
|\nabla u|^{p-2} \frac{\partial u}{\partial \nu}=F_{u}(x, u, v), \quad|\nabla u|^{q-2} \frac{\partial v}{\partial \nu}=F_{v}(x, u, v)
$$

The proofs are done under suitable assumptions on the potential $F$, and based on variational arguments. Our results include subcritical, resonant and critical growth on $F$.

2000 Mathematics Subject Classification: 35J65, 35J20, 35J55.

Key words: elliptic systems, nonlinear boundary conditions, variational problems.

Supported by Univ. de Buenos Aires grant TX48, by ANPCyT PICT No. 03-00137 and by Fundación Antorchas. JFB is also supported by ANPCyT PICT No. 03-10608. JFB and JDR are members of CONICET. 


\section{Introduction}

In this paper we study the existence of nontrivial solutions of the quasilinear elliptic system

$$
\Delta_{p} u=|u|^{p-2} u, \quad \Delta_{q} v=|v|^{q-2} v \quad \text { in } \Omega
$$

with nonlinear coupling at the boundary given by

$$
|\nabla u|^{p-2} \frac{\partial u}{\partial \nu}=F_{u}(x, u, v), \quad|\nabla v|^{q-2} \frac{\partial v}{\partial \nu}=F_{v}(x, u, v) \quad \text { on } \partial \Omega \text {. }
$$

Here $\Omega$ is a bounded domain in $\mathbb{R}^{N}$ with smooth boundary, $\Delta_{p} u=\operatorname{div}\left(|\nabla u|^{p-2} \nabla u\right)$ is the $p$-Laplacian, $\frac{\partial}{\partial \nu}$ is the outer normal derivative and $\left(F_{u}, F_{v}\right)$ is the gradient of some positive potential $F: \partial \Omega \times \mathbb{R} \times \mathbb{R} \rightarrow \mathbb{R}$ with precise hypotheses that we state below.

Existence results for nonlinear elliptic systems have deserved a great deal of interest in recent years, in particular when the nonlinear term appears as a source in the equation complemented with Dirichlet boundary conditions. For this type of result see, among others, $[2,3,7,9,10,11]$ and the survey [8]. There are two major classes of systems that can be treated variationally: Hamiltonian and gradient systems. Here we deal with a gradient problem. The Hamiltonian case (for $p=q=2$ ) was analyzed in $[13,14,22]$. Problems with no variational structure can be treated via fixed-point arguments. For example (1.1) with nonlinear boundary conditions, $p=q=2$ and without variational assumptions has been studied in [15].

For (weak) solutions of (1.1)-(1.2) we understand critical points of the functional

$$
\mathcal{F}(u, v)=\int_{\Omega} \frac{|\nabla u|^{p}}{p}+\frac{|u|^{p}}{p} d x+\int_{\Omega} \frac{|\nabla v|^{q}}{q}+\frac{|v|^{q}}{q} d x-\int_{\partial \Omega} F(x, u, v) d \sigma .
$$

where $d \sigma$ is the boundary measure.

The geometry of $\mathcal{F}$ is similar to the one of the functional

$$
\mathcal{F}_{1}(u)=\frac{1}{p} \int_{\Omega}|\nabla u|^{p}+|u|^{p} d x-\int_{\partial \Omega} F(x, u) d \sigma
$$

which corresponds to a single quasilinear equation with nonlinear boundary conditions. The functional $\mathcal{F}_{1}$ was studied in [16] where essentially the case $F(x, u)=$ $|u|^{r}$ was consider. Recently, in [12], the functional $\mathcal{F}_{1}$ was studied where the potential $F$ is allowed to change sign.

However, some interesting phenomena appear in (1.3) due to the coupling in the system (1.1)-(1.2). Our results for (1.1)-(1.2) generalize the ones in [16] both to systems and to more general potentials. 
In [2] the functional

$$
\overline{\mathcal{F}}(u, v)=\int_{\Omega}|\nabla u|^{p}+|\nabla v|^{q} d x-\int_{\Omega} \bar{F}(x, u, v) d x
$$

was analyzed. In this paper we extend their results to the nonlinear boundary condition case and moreover some new results are obtained. For instance, multiplicity results in the subcritical case with an oddness condition on $F$ and, mainly, existence results with critical growth.

Let us introduce the precise assumptions of $F$. From now on, we fix $1<$ $p, q<N$, and so the functional $\mathcal{F}$ will be defined in the Banach space $W^{1, p}(\Omega) \times$ $W^{1, q}(\Omega)$. Of course, the growth of $F$ has to be controlled in order for $\mathcal{F}$ to make sense for $(u, v) \in W^{1, p}(\Omega) \times W^{1, q}(\Omega)$. According to the Sobolev trace embedding, we impose

$$
|F(x, u, v)| \leq C\left(1+|u|^{p_{*}}+|v|^{q_{*}}\right),
$$

where $p_{*}=p(N-1) /(N-p)$ and $q_{*}=q(N-1) /(N-q)$ are the critical Sobolev trace exponents and $C$ is some positive constant. With $\left(\mathrm{F}_{1}\right)$, as $W^{1, p}(\Omega) \rightarrow$ $L^{p_{*}}(\partial \Omega)$ and $W^{1, q}(\Omega) \rightarrow L^{q_{*}}(\partial \Omega)$ by the Sobolev trace Theorem, we have that $\mathcal{F}$ is well defined.

In order to apply variational techniques, we need the functional $\mathcal{F}$ to be $C^{1}$. To this end, $\left(F_{1}\right)$ is not enough. One has to consider the stronger assumption

$$
\begin{aligned}
& \left|F_{u}(x, u, v)\right| \leq C\left(1+|u|^{p_{*}-1}+|v|^{\frac{q_{*}\left(p_{*}-1\right)}{p_{*}}}\right) \\
& \left|F_{v}(x, u, v)\right| \leq C\left(1+|v|^{q_{*}-1}+|u|^{\frac{p_{*}\left(q_{*}-1\right)}{q_{*}}}\right) .
\end{aligned}
$$

One can easily check that $\left(\mathrm{F}_{2}\right)$ implies $\left(\mathrm{F}_{1}\right)$ and under $\left(\mathrm{F}_{2}\right)$, it follows that critical points of $\mathcal{F}$ are weak solutions of (1.1)-(1.2).

Now, the geometry of $\mathcal{F}$ depends strongly on the precise growth of the potential $F$. That is, on the exponents $r$ and $s$ in the inequality

$$
|F(x, u, v)| \leq C\left(1+|u|^{r}+|v|^{s}\right),
$$

where $r \leq p_{*}$ and $s \leq q_{*}$.

We will distinguish mainly four different cases:

(1) $r<p$ and $s<q$. (Sublinear-like)

(2) $r=p$ and $s=q$. (Resonant)

(3) $p<r<p_{*}$ and $q<s<q_{*}$. (Superlinear-like, subcritical)

(4) $r=p_{*}$ and $s=q_{*}$. (Critical)

Of course, the case of interest is

$$
F(x, 0,0)=F_{u}(x, 0,0)=F_{v}(x, 0,0)=0, \quad \text { for } x \in \partial \Omega,
$$

then $u \equiv v \equiv 0$ is a trivial solution of the system (1.1)-(1.2). 
First, we turn our attention to the superlinear and subcritical case (3).

In order to verify the Palais-Smale condition, we need to impose the following assumption: There exist $R>0, \theta_{p}$ and $\theta_{q}$ with

$$
\theta_{p}<\frac{1}{p}, \quad \theta_{q}<\frac{1}{q}
$$

such that

$$
0<F(x, u, v) \leq \theta_{p} u F_{u}(x, u, v)+\theta_{q} v F_{v}(x, u, v)
$$

for all $x \in \partial \Omega$ and $|u|,|v| \geq R$.

We have,

Theorem 1.1 Assume that the potential $F$ satisfies $\left(\mathrm{F}_{2}\right),\left(\mathrm{F}_{3}\right)$ with $r$, s as in (3), $\left(\mathrm{F}_{4}\right)$ and $\left(\mathrm{F}_{5}\right)$. Assume moreover that there exists constants $c>0$ and $\varepsilon>0$ and $p_{*}>\bar{r}>p, q_{*}>\bar{s}>q$ such that

$$
|F(x, u, v)| \leq c\left(|u|^{\bar{r}}+|v|^{\bar{s}}\right), \quad \text { for } x \in \partial \Omega,|u|,|v| \leq \varepsilon .
$$

Then $\mathcal{F}$ has a critical point. If, moreover, $F$ is even then $\mathcal{F}$ has infinitely many critical points which are unbounded in $W^{1, p}(\Omega) \times W^{1, q}(\Omega)$.

Case (1) is similar in nature to a sublinear problem for the usual Laplacian. So, direct minimization yields a nontrivial solution. However, under a hypothesis similar to $\left(\mathrm{F}_{5}\right)$ we can show the existence of infinitely many solutions (of course, with an oddness assumption on $F$ ). The condition is: There exists $R>0, \theta_{p}$ and $\theta_{q}$ with

$$
\theta_{p}<\frac{1}{p}, \quad \theta_{q}<\frac{1}{q}
$$

such that

$$
\theta_{p} u F_{u}(x, u, v)+\theta_{q} v F_{v}(x, u, v)-F(x, u, v) \geq-c\left(|u|^{r}+|v|^{s}\right),
$$

for all $x \in \partial \Omega$ and $|u|,|v| \geq R$.

We have the following.

Theorem 1.2 Assume that the potential $F$ satisfies $\left(\mathrm{F}_{2}\right),\left(\mathrm{F}_{3}\right)$ with $r, s$ as in $(1)$ and $\left(\mathrm{F}_{4}\right)$. Then $\mathcal{F}$ has a nontrivial critical point providing there exists a constant $R>0, \theta<1$ and a continuous function $K: \partial \Omega \times \mathbb{R} \times \mathbb{R} \rightarrow \mathbb{R}$ such that

$$
F\left(x, t^{\frac{1}{p}} u, t^{\frac{1}{q}} v\right) \geq t^{\theta} K(x, u, v), \quad \text { for } x \in \partial \Omega,|u|,|v| \leq R, \text { and small } t>0 . \quad\left(\mathrm{F}_{8}\right)
$$

If, moreover, $F$ is even and $\left(\mathrm{F}_{7}\right)$ holds, then $\mathcal{F}$ has infinitely many critical points which form a compact set in $W^{1, p}(\Omega) \times W^{1, q}(\Omega)$. 
The case (2) is a resonant problem. So there is an underlying (nonlinear) eigenvalue problem. In this case, it is natural to assume a condition on $F$ that implies that the functional $\mathcal{F}$ satisfies the so-called Cerami condition (see (5.5)). This assumption is, there are positive constants $c, R, a, b$ with $0<a<p, 0<b<q$ such that

$$
\frac{1}{p} u F_{u}(x, u, v)+\frac{1}{q} v F_{v}(x, u, v)-F(x, u, v) \geq c\left(|u|^{a}+|v|^{b}\right),
$$

for $x \in \partial \Omega,|u|,|v|>R$. This type of condition was introduced, for the Dirichlet boundary condition case, by $[5,6]$.

In order to avoid resonance, we need to understand the underlying eigenvalue problem. A similar eigenvalue problem for the Dirichlet boundary condition case, was introduced in [2]. Let $G: \mathbb{R}^{2} \rightarrow[0, \infty)$ be a $C^{1}$ positive even function such that

$$
\begin{aligned}
& G\left(t^{\frac{1}{p}} u, t^{\frac{1}{q}} v\right)=t G(u, v) . \\
& G(u, v) \leq k\left(|u|^{p}+|v|^{q}\right)
\end{aligned}
$$

The eigenvalue problem is

$$
\begin{aligned}
& \Delta_{p} u=|u|^{p-2} u \\
& \Delta_{q} v=|v|^{q-2} v, \quad \text { in } \Omega \\
& |\nabla u|^{p-2} \frac{\partial u}{\partial \nu}-a G_{u}=\lambda|u|^{p-2} u,|\nabla v|^{q-2} \frac{\partial v}{\partial \nu}-a G_{v}=\lambda|v|^{q-2} v, \text { on } \partial \Omega,
\end{aligned}
$$

where $a \in L^{\infty}(\partial \Omega)$.

We will see that problem (1.4) has a first eigenvalue $\lambda_{1}(a)$. So in order to avoid resonance, we assume that there exists positive numbers $R$ and $\varepsilon$, and $a, b \in L^{\infty}(\partial \Omega)$ such that

$$
\begin{array}{lll}
\lambda_{1}(a)<0, & F(x, u, v) \geq a(x) G(u, v), & |u|,|v| \geq R, \\
\lambda_{1}(b)>0, & F(x, u, v) \leq b(x) \bar{G}(u, v), & |u|,|v| \leq \varepsilon,
\end{array}
$$

where $G$ and $\bar{G}$ satisfy $\left(\mathrm{G}_{1}\right)$.

We have the following,

Theorem 1.3 Assume that the potential $F$ satisfies $\left(\mathrm{F}_{2}\right),\left(\mathrm{F}_{3}\right)$ with $r$ and $s$ as in (2), $\left(\mathrm{F}_{4}\right),\left(\mathrm{F}_{9}\right)$ and $\left(\mathrm{F}_{10}\right)$. Then the functional $\mathcal{F}$ has a nontrivial critical point.

Now we turn our attention to the critical case (4). As it is well known, the compactness in the immersion $W^{1, p}(\Omega) \times W^{1, q}(\Omega) \rightarrow L^{p_{*}}(\partial \Omega) \times L^{q_{*}}(\partial \Omega)$ fails, so the functional $\mathcal{F}$ does not verify the Palais-Smale condition. However, by applying the compensated compactness method (see $[18,19]$ ), we can prove that $\mathcal{F}$ satisfy a local Palais-Smale condition that will suffices to apply the usual variational techniques. 
The hypotheses on the potential $F$ in order to apply the compensated compactness method are

$$
F(x, u, v):=F_{\lambda}(x, u, v):=F^{c}(x, u, v)+\lambda F^{s}(x, u, v)
$$

where $F^{c}$ is the critical part of $F_{\lambda}$ and $F^{s}$ is a subcritical perturbation, that verifies $\left(\mathrm{F}_{3}\right)$ with $r$ and $s$ as in (1) or (3).

The hypotheses on the critical part $F^{c}$ are: there exist two constants $c, C>0$ such that

$$
\begin{aligned}
& c\left(|u|^{p_{*}}+|v|^{q_{*}}\right) \leq F_{u}^{c}(x, u, v) u+F_{v}^{c}(x, u, v) v \leq C\left(|u|^{p_{*}}+|v|^{q_{*}}\right) . \\
& c\left(|u|^{p_{*}}+|v|^{q_{*}}\right) \leq \theta_{p} F_{u}^{c}(x, u, v) u+\theta_{q} F_{v}^{c}(x, u, v) v-F^{c}(x, u, v),
\end{aligned}
$$

where $\theta_{p}$ and $\theta_{q}$ are defined for the two cases in $\left(\mathrm{F}_{5}\right)$ and $\left(\mathrm{F}_{7}\right)$.

For the subcritical perturbation $F^{s}$, we need also to impose the following condition,

$$
F_{u}^{s}(x, u, v) u+F_{v}^{s}(x, u, v) v \leq C\left(1+|u|^{r}+|v|^{s}\right) .
$$

We have the following theorem.

Theorem 1.4 Assume that $F_{\lambda}$ satisfies $\left(\mathrm{F}_{11}\right)$ with $F^{s}$ satisfying $\left(\mathrm{F}_{2}\right),\left(\mathrm{F}_{3}\right)$ with $r$ and $s$ as in (3), $\left(\mathrm{F}_{4}\right),\left(\mathrm{F}_{5}\right)$ and $\left(\mathrm{F}_{1}^{s}\right)$ and $F^{c}$ satisfying $\left(\mathrm{F}_{1}^{c}\right)$ and $\left(\mathrm{F}_{2}^{c}\right)$. Then there exists a constant $\bar{\Lambda}>0$ such that, if $\lambda>\bar{\Lambda}, \mathcal{F}$ has a critical point in $W^{1, p}(\Omega) \times W^{1, q}(\Omega)$

Finally, for sublinear perturbations we have,

Theorem 1.5 Assume that $F_{\lambda}$ satisfies $\left(\mathrm{F}_{11}\right)$ with $F^{s}$ satisfying $\left(\mathrm{F}_{2}\right),\left(\mathrm{F}_{3}\right)$ with $r$ and $s$ as in $(1),\left(\mathrm{F}_{4}\right),\left(\mathrm{F}_{7}\right)$ and $\left(\mathrm{F}_{1}^{s}\right)$ and $F^{c}$ satisfying $\left(\mathrm{F}_{1}^{c}\right)$ and $\left(\mathrm{F}_{2}^{c}\right)$. Then there exists a constant $\Lambda>0$ such that, if $0<\lambda<\Lambda, \mathcal{F}$ has a nontrivial critical point. Moreover, if $F$ is even then $\mathcal{F}$ has infinitely many critical points in $W^{1, p}(\Omega) \times$ $W^{1, q}(\Omega)$.

The rest of the paper is organized as follows: In section 2 we show some classes of potentials that verifies our hypotheses in each case. In section 3 , we deal with the superlinear-like and subcritical case (Theorem 1.1). In section 4, we analyze the sublinear-like case (Theorem 1.2). Then, in section 5 we study the resonant case (Theorem 1.3) and also the eigenvalue problem (1.4). Finally, in the last two sections, section 6 and section 7, we deal with the critical cases (Theorem 1.4 and Theorem 1.5 respectively). 


\section{Examples of potentials}

In this section we exhibit examples of potentials that fulfil our hypotheses. Most of our examples are borrowed from [2].

Example 2.1 $F(x, u, v)=a(x)|u|^{r}+b(x)|v|^{s}$, where $a, b \in L^{\infty}(\partial \Omega)$ are positive. Of course, these potentials are of little interest because they give rise to two uncoupled PDEs.

Example 2.2 $F(x, u, v)=a(x)|u|^{\alpha}|v|^{\beta}$, where $a \in L^{\infty}(\partial \Omega)$ is positive. This potential verifies $\left(\mathrm{F}_{1}\right)-\left(\mathrm{F}_{4}\right)$ if

$$
\frac{\alpha}{p_{*}}+\frac{\beta}{q_{*}} \leq 1 .
$$

Now, if we have strict inequality, we are in the subcritical cases (1)-(3). To avoid resonance, we need

$$
\frac{\alpha}{r}+\frac{\beta}{s}=1
$$

where $r \neq p$ and $s \neq q$. In this case the hypotheses $\left(\mathrm{F}_{1}\right)-\left(\mathrm{F}_{8}\right)$ are satisfied. Theorem 1.1 and Theorem 1.2 hold true for these potentials.

Example 2.3 $F(x, u, v)=c(x)|u|^{p}+d(x)|v|^{q}+e(x)|u|^{\alpha}|v|^{\beta}$, where $c, d, e \in L^{\infty}(\partial \Omega)$ are positive functions and

$$
\frac{\alpha}{p}+\frac{\beta}{q}=1
$$

In this case, hypotheses $\left(\mathrm{F}_{1}\right)-\left(\mathrm{F}_{4}\right)$ and $\left(\mathrm{F}_{9}\right)$ are satisfied. To see that $\left(\mathrm{F}_{10}\right)$ is also verified, we can take $G(u, v)=\bar{G}(u, v)=\frac{1}{p}|u|^{p}+\frac{1}{q}|v|^{q}$ and $a(x)=-M, b(x)=M$ where $M$ is a (large) positive number. Then $\lambda_{1}(a)=\min \left\{\lambda_{1, p}, \lambda_{1, q}\right\}-M$ and $\lambda_{1}(b)=\min \left\{\lambda_{1, p}, \lambda_{1, q}\right\}+M$ where $\lambda_{1, p}$ is the first eigenvalue of

$$
\begin{cases}\Delta_{p} u=|u|^{p-2} u & \text { in } \Omega \\ |\nabla u|^{p-2} \frac{\partial u}{\partial \nu}=\lambda|u|^{p-2} u & \text { on } \partial \Omega .\end{cases}
$$

For properties of this eigenvalue problem we refer to $[16,20]$.

Example 2.4 $F(x, u, v)=F_{\lambda}(x, u, v)=F^{c}(x, u, v)+\lambda F^{s}(x, u, v)$ and $F^{s}(x, u, v)$ is as in Example 2.2 and $F^{c}(x, u, v)=b(x)|u|^{p_{*}}+c(x)|v|^{q_{*}}+d(x)|u|^{\alpha}|v|^{\beta}$, where $b, c, d \in L^{\infty}(\partial \Omega)$ are positive functions and

$$
\frac{\alpha}{p_{*}}+\frac{\beta}{q_{*}}=1 .
$$

It is now easy to check that $F_{\lambda}$ verifies our hypotheses needed in Theorems 1.4 and 1.5 , provided that the perturbation $F^{s}$ is either sublinear-like or either superlinearlike and subcritical. 


\section{Superlinear and subcritical case. $p<r<p_{*}$ and $q<s<q_{*}$}

The following Lemma, that will be helpful in order to prove the Palais-Smale condition, was proved in [16].

Lemma 3.1 [[16], Lemma 2.1]. Let $\phi \in W^{1, p}(\Omega)^{\prime}$ (the topological dual space of $\left.W^{1, p}(\Omega)\right)$. Then there exists a unique weak solution $u \in W^{1, p}(\Omega)$ of

$$
-\Delta_{p} u+|u|^{p-2} u=\phi
$$

Moreover, the operator $A_{p}: \phi \mapsto u$ is continuous.

With this Lemma we can verify the Palais-Smale condition for $\mathcal{F}$.

Lemma 3.2 The functional $\mathcal{F}$ satisfies the Palais-Smale condition.

Proof. Let $\left(u_{k}, v_{k}\right)_{k \geq 1} \subset W^{1, p}(\Omega) \times W^{1, q}(\Omega)$ be a Palais-Smale sequence, that is a sequence such that

$$
\mathcal{F}\left(u_{k}, v_{k}\right) \rightarrow c \quad \text { and } \quad \mathcal{F}^{\prime}\left(u_{k}, v_{k}\right) \rightarrow 0
$$

Let us first prove that (3.1) implies that $\left(u_{k}, v_{k}\right)$ is bounded. From (3.1) it follows that there exists a sequence $\varepsilon_{k} \rightarrow 0$ such that

$\left|\mathcal{F}^{\prime}\left(u_{k}, v_{k}\right)(w, z)\right| \leq \varepsilon_{k}\left(\|w\|_{W^{1, p}(\Omega)}+\|z\|_{W^{1, q}(\Omega)}\right), \quad \forall(w, z) \in W^{1, p}(\Omega) \times W^{1, q}(\Omega)$.

Now we have, by $\left(\mathrm{F}_{5}\right)$,

$$
\begin{aligned}
c+1 \geq & \mathcal{F}\left(u_{k}, v_{k}\right)-\mathcal{F}^{\prime}\left(u_{k}, v_{k}\right)\left(\theta_{p} u_{k}, \theta_{q} v_{k}\right)+\mathcal{F}^{\prime}\left(u_{k}, v_{k}\right)\left(\theta_{p} u_{k}, \theta_{q} v_{k}\right) \\
\geq & \left(\frac{1}{p}-\theta_{p}\right)\left\|u_{k}\right\|_{W^{1, p}(\Omega)}^{p}+\left(\frac{1}{q}-\theta_{q}\right)\left\|v_{k}\right\|_{W^{1, q}(\Omega)}^{q}+\mathcal{F}^{\prime}\left(u_{k}, v_{k}\right)\left(\theta_{p} u_{k}, \theta_{q} v_{k}\right) \\
\geq & \left(\frac{1}{p}-\theta_{p}\right)\left\|u_{k}\right\|_{W^{1, p}(\Omega)}^{p}+\left(\frac{1}{q}-\theta_{q}\right)\left\|v_{k}\right\|_{W^{1, q}(\Omega)}^{q} \\
& -\varepsilon_{k}\left(\theta_{p}\left\|u_{k}\right\|_{W^{1, p}(\Omega)}+\theta_{q}\left\|v_{k}\right\|_{W^{1, q}(\Omega)}\right)
\end{aligned}
$$

hence, $\left(u_{k}, v_{k}\right)$ is bounded in $W^{1, p}(\Omega) \times W^{1, q}(\Omega)$.

By compactness we can assume that $\left(u_{k}, v_{k}\right) \rightarrow(u, v)$ weakly in $W^{1, p}(\Omega) \times$ $W^{1, q}(\Omega)$ and $\left(u_{k}, v_{k}\right) \rightarrow(u, v)$ strongly in $L^{r}(\partial \Omega) \times L^{s}(\partial \Omega)$ and a.e. in $\partial \Omega$. Then, as $p<r<p_{*}$ and $q<s<q_{*}$, it follows that, $F_{u}\left(x, u_{k}, v_{k}\right) \rightarrow F_{u}(x, u, v)$ in $L^{p_{*}^{\prime}}(\partial \Omega)$ and $F_{v}\left(x, u_{k}, v_{k}\right) \rightarrow F_{v}(x, u, v)$ in $L^{q_{*}^{\prime}}(\partial \Omega)$ and hence in $W^{1, p}(\Omega)^{\prime} \times$ $W^{1, q}(\Omega)^{\prime}$. Therefore, according to Lemma 3.1,

$$
\left(u_{k}, v_{k}\right) \rightarrow\left(A_{p}\left(F_{u}(x, u, v)\right), A_{q}\left(F_{v}(x, u, v)\right)\right), \quad \text { in } W^{1, p}(\Omega) \times W^{1, q}(\Omega) .
$$

This completes the proof. 
Proof of Theorem 1.1. Existence part. The fact that $\mathcal{F}$ is $C^{1}$ is a straightforward adaptation of the results in [21]. The Palais-Smale condition was already checked in Lemma 3.2 .

Hypotheses $\left(\mathrm{F}_{3}\right),\left(\mathrm{F}_{4}\right),\left(\mathrm{F}_{6}\right)$ guarantee that we are in the geometrical assumptions needed to apply the Mountain Pass Lemma (see [21]). In fact, from the Sobolev immersion theorem, we obtain

$$
\begin{aligned}
\mathcal{F}(u, v) & =\frac{1}{p}\|u\|_{W^{1, p}(\Omega)}^{p}+\frac{1}{q}\|v\|_{W^{1, q}(\Omega)}^{q}-\int_{\partial \Omega} F(x, u, v) d \sigma \\
& \geq \frac{1}{p}\|u\|_{W^{1, p}(\Omega)}^{p}+\frac{1}{q}\|v\|_{W^{1, q}(\Omega)}^{q}-C\left(1+\|u\|_{W^{1, p}(\Omega)}^{r}+\|v\|_{W^{1, q}(\Omega)}^{s}\right) \\
& =g\left(\|u\|_{W^{1, p}(\Omega)},\|v\|_{W^{1, q}(\Omega)}\right)
\end{aligned}
$$

where $g(x, y)=\frac{1}{p} x^{p}+\frac{1}{q} y^{q}-C\left(x^{\bar{r}}+y^{\bar{s}}\right)$. As $\bar{r}>p$ and $\bar{s}>q, \mathcal{F}(u, v)>0$ in $0<$ $\|u\|_{W^{1, p}(\Omega)}+\|v\|_{W^{1, q}(\Omega)}<\rho$ and $\mathcal{F}(u, v) \geq c>0$ on $\|u\|_{W^{1, p}(\Omega)}+\|v\|_{W^{1, q}(\Omega)}=\rho$.

As in [2], one can check that $\left(\mathrm{F}_{5}\right)$ implies that there exists a positive function $K(x, u, v)$ such that

$$
F\left(x, t^{\theta_{p}} u, t^{\theta_{q}} v\right) \geq t K(x, u, v)
$$

from where it follows that there exists $\left(u_{0}, v_{0}\right) \in W^{1, p}(\Omega) \times W^{1, q}(\Omega)$ with $\mathcal{F}\left(u_{0}, v_{0}\right)<0$. Therefore we have a nontrivial critical point of $\mathcal{F}$.

Now, in order to prove the multiplicity result when $\mathcal{F}$ is even, we introduce a topological tool, the genus, that was introduced in [17] but we will use an equivalent definition due to [4]. Given a Banach Space $X$, we consider the class

$$
\Sigma=\{A \subset X: A \text { is closed, } A=-A\} .
$$

Over this class we define the genus, $\gamma: \Sigma \rightarrow \mathbb{N} \cup\{\infty\}$, as

$\gamma(A)=\min \left\{k \in \mathbb{N}:\right.$ there exists $\left.\varphi \in C\left(A, \mathbb{R}^{k}-\{0\}\right), \varphi(x)=-\varphi(-x)\right\}$.

We will use the following Theorem whose proof can be found in [1],

Theorem 3.1 ([1, Theorem 2.23]). Let $\mathcal{F}: X \rightarrow \mathbb{R}$ verifying

(1) $\mathcal{F} \in C^{1}(X)$ and even.

(2) $\mathcal{F}$ verifies the Palais-Smale condition.

(3) There exists a constant $r>0$ such that $\mathcal{F}(u)>0$ in $0<\|u\|_{X}<r$, and $\mathcal{F}(u) \geq c>0$ if $\|u\|_{X}=r$.

(4) There exists a closed subspace $E_{m} \subset X$ of dimension $m$, and a compact set $A_{m} \subset E_{m}$ such that $\mathcal{F}<0$ on $A_{m}$ and 0 lies in a bounded component of $E_{m}-A_{m}$ in $E_{m}$. 
Let $B$ be the unit ball in $X$, we define

$\Gamma=\{h \in C(X, X): h(0)=0, h$ is an odd homeomorphism and $\mathcal{F}(h(B)) \geq 0\}$,

and

$\mathcal{K}_{m}=\{K \subset X: K=-K, K$ is compact, and $\gamma(K \cap h(\partial B)) \geq m$ for all $h \in \Gamma\}$

Then,

$$
c_{m}=\inf _{K \in \mathcal{K}_{m}} \max _{u \in K} \mathcal{F}(u)
$$

is a critical value of $\mathcal{F}$, with $0<c \leq c_{m} \leq c_{m+1}<\infty$. Moreover, if $c_{m}=c_{m+1}=$ $\cdots=c_{m+r}$ then $\gamma\left(K_{c_{m}}\right) \geq r+1$ where $K_{c_{m}}=\left\{u \in X: \mathcal{F}^{\prime}(u)=0, \mathcal{F}(u)=c_{m}\right\}$.

End of the proof of Theorem 1.1. We need to check the hypotheses of Theorem 3.1. Hypothesis (1) is a direct consequence of the oddness assumption on $F$. Hypotheses (2) and (3) were already verified. Finally, to verify (4), let us consider a sequence of subspaces $E_{m} \subset W^{1, p}(\Omega) \times W^{1, q}(\Omega)$ of dimension $m$ such that $E_{m} \subset E_{m+1}$ and $|u|,|v| \not \equiv 0$ on $\partial \Omega$ for $(u, v) \neq(0,0),(u, v) \in E_{m}$. Hence,

$$
\min _{(u, v) \in B_{m}} \int_{\partial \Omega} K(x, u, v) d \sigma>0
$$

where $B_{m}=\left\{(u, v) \in E_{m}: \int_{\partial \Omega} K(x, u, v) d \sigma=1\right\}$. Now we observe that, using $(3.2)$,

$$
\begin{aligned}
\mathcal{F}\left(t^{\theta_{p}} u, t^{\theta_{q}} v\right) & \leq \frac{t^{\theta_{p} p}}{p}\|u\|_{W^{1, p}(\Omega)}^{p}+\frac{t^{\theta_{q} q}}{q}\|v\|_{W^{1, q}(\Omega)}^{q}-\min _{(u, v) \in B_{m}} \int_{\partial \Omega} F\left(x, t^{\theta_{p}} u, t^{\theta_{q}} v\right) d \sigma \\
& \leq \frac{t^{\theta_{p} p}}{p}\|u\|_{W^{1, p}(\Omega)}^{p}+\frac{t^{\theta_{q} q}}{q}\|v\|_{W^{1, q}(\Omega)}^{q}-C t
\end{aligned}
$$

for all $(u, v) \in B_{m}$, and then for $t \geq t_{0}$, (4) follows by taking $A_{m}=t_{0} B_{m}$.

To end this section, let us see that the critical points of $\mathcal{F}$ that we have found are unbounded in $W^{1, p}(\Omega) \times W^{1, q}(\Omega)$. The arguments follow closely the ones given in [16]. We only sketch them for the convenience of the reader.

The result will follow from the next lemma,

Lemma 3.3 Let $\left(c_{m}\right) \subset \mathbb{R}$ be the sequence of critical values given by Theorem 3.1. Then $\lim _{m \rightarrow \infty} c_{m}=\infty$.

Proof. Let

$$
\begin{aligned}
M=\{ & (u, v) \in W^{1, p}(\Omega) \times W^{1, q}(\Omega): u, v \neq 0, \\
& \left.\frac{1}{\lambda p}\|u\|_{W^{1, p}(\Omega)}^{p} \leq\|u\|_{L^{r}(\partial \Omega)}^{r}, \frac{1}{\lambda q}\|v\|_{W^{1, q}(\Omega)}^{q} \leq\|v\|_{L^{s}(\partial \Omega)}^{s}\right\} .
\end{aligned}
$$


By the Sobolev trace Theorem, there exists a constant $\theta>0$ such that

$$
\theta<\|u\|_{L^{r}(\partial \Omega)}^{r}, \theta<\|v\|_{L^{s}(\partial \Omega)}^{s} \quad \forall(u, v) \in M .
$$

Let us define

$$
b_{m}=\sup _{h \in \Gamma} \inf _{\left\{(u, v) \in \partial B \cap E_{m-1}^{c}\right\}} \mathcal{F}(h(u, v)) .
$$

It is proved in [1] that $b_{m} \leq c_{m}$, hence to prove our result it is enough to show that $b_{m} \rightarrow \infty$.

Now, $b_{m+1} \geq \inf _{\left\{(u, v) \in \partial B \cap E_{m}^{c}\right\}} \mathcal{F}(h(u, v))$ for all $h \in \Gamma$. Using (3.3) and following [16] one can construct $\tilde{h}_{m} \in \Gamma$ such that $\lim _{m \rightarrow \infty} \inf _{\left\{(u, v) \in \partial B \cap E_{m}^{c}\right\}}$ $\mathcal{F}\left(\tilde{h}_{m}(u, v)\right)=\infty$. This ends the proof of the lemma.

\section{Sublinear case. $r<p$ and $s<q$}

In this case, a nontrivial solution can be found easily by direct minimization.

Proof of Theorem 1.2. Existence part. By a standard compactness argument, an absolute minimizer of $\mathcal{F}$ in $W^{1, p}(\Omega) \times W^{1, q}(\Omega)$ exists. We need to show that this minimizer is nontrivial. In fact, let $(u, v) \in W^{1, p}(\Omega) \times W^{1, q}(\Omega)$ such that $K(x, u, v) \neq 0$. Then, by $\left(\mathrm{F}_{8}\right)$,

$$
\mathcal{F}\left(t^{1 / p} u, t^{1 / q} v\right) \geq t\left(\|u\|_{W^{1, p}(\Omega)}^{p}+\|v\|_{W^{1, q}(\Omega)}^{q}\right)-t^{\theta} \int_{\partial \Omega} K(x, u, v) d \sigma .
$$

Then, taking $t$ small enough we obtain $\inf \mathcal{F}<0$.

For the multiplicity part, we begin by showing that the Palais-Smale condition still holds.

Lemma 4.1 The functional $\mathcal{F}$ is bounded below and verifies the Palais-Smale condition.

Proof. First, by the Sobolev-trace inequality and $\left(\mathrm{F}_{3}\right)$, we have

$$
\begin{aligned}
\mathcal{F}(u, v) \geq \frac{1}{p}\|u\|_{W^{1, p}(\Omega)}^{p} & +\frac{1}{q}\|v\|_{W^{1, q}(\Omega)}^{q}-C\left(1+\|u\|_{W^{1, p}(\Omega)}^{r}+\|v\|_{W^{1, q}(\Omega)}^{s}\right) \\
& \equiv h\left(\|u\|_{W^{1, p}(\Omega)},\|v\|_{W^{1, q}(\Omega)}\right)
\end{aligned}
$$

where

$$
h(a, b)=\frac{1}{p} a^{p}+\frac{1}{p} b^{q}-C\left(1+a^{r}+b^{s}\right) .
$$

As $r<p$ and $s<q, h(a, b)$ is bounded below and therefore we conclude that $\mathcal{F}$ is bounded below. 
Now to prove the Palais-Smale condition, let $\left(u_{k}, v_{k}\right) \in W^{1, p}(\Omega) \times W^{1, q}(\Omega)$ a Palais-Smale sequence. As $c=\lim _{k \rightarrow \infty} \mathcal{F}\left(u_{k}, v_{k}\right)$, using that

$$
\left|\mathcal{F}^{\prime}\left(u_{k}, v_{k}\right)(w, z)\right| \leq \varepsilon_{k}\left(\|w\|_{W^{1, p}(\Omega)}+\|z\|_{W^{1, q}(\Omega)}\right)
$$

where $\varepsilon_{k} \rightarrow 0$ in $W^{1, p}(\Omega)^{\prime} \times W^{1, q}(\Omega)^{\prime}$ and $\left(\mathrm{F}_{7}\right)$ we have that, for $k$ large enough,

$$
\begin{aligned}
c+1 \geq & \mathcal{F}\left(u_{k}, v_{k}\right)-\mathcal{F}^{\prime}\left(u_{k}, v_{k}\right)\left(\theta_{p} u_{k}, \theta_{q} v_{k}\right)+\mathcal{F}^{\prime}\left(u_{k}, v_{k}\right)\left(\theta_{p} u_{k}, \theta_{q} v_{k}\right) \\
\geq & \left(\frac{1}{p}-\theta_{p}\right)\left\|u_{k}\right\|_{W^{1, p}(\Omega)}^{p}+\left(\frac{1}{q}-\theta_{q}\right)\left\|v_{k}\right\|_{W^{1, q}(\Omega)}^{q} \\
& -C\left(\left\|u_{k}\right\|_{W^{1, p}(\Omega)}^{r}+\left\|v_{k}\right\|_{W^{1, q}(\Omega)}^{s}\right)+\mathcal{F}^{\prime}\left(u_{k}, v_{k}\right)\left(\theta_{p} u_{k}, \theta_{q} v_{k}\right) \\
\geq & \left(\frac{1}{p}-\theta_{p}\right)\left\|u_{k}\right\|_{W^{1, p}(\Omega)}^{p}+\left(\frac{1}{q}-\theta_{q}\right)\left\|v_{k}\right\|_{W^{1, q}(\Omega)}^{q} \\
& -C\left(\left\|u_{k}\right\|_{W^{1, p}(\Omega)}^{r}+\left\|v_{k}\right\|_{W^{1, q}(\Omega)}^{s}\right)-\varepsilon_{k}\left(\theta_{p}\left\|u_{k}\right\|_{W^{1, p}(\Omega)}+\theta_{q}\left\|v_{k}\right\|_{W^{1, q}(\Omega)}\right)
\end{aligned}
$$

and using that $r<p$ and $s<q$ it follows that $\left\|u_{k}\right\|_{W^{1, p}(\Omega)} \leq C$ and $\left\|v_{k}\right\|_{W^{1, q}(\Omega)} \leq$ $C$. Therefore, the result follows as in Lemma 3.2.

Now we need the following lemma.

Lemma 4.2 For every $n \in \mathbb{N}$ there exists a constant $\varepsilon>0$ such that

$$
\gamma\left(\mathcal{F}^{-\varepsilon}\right) \geq n
$$

where $\mathcal{F}^{c}=\left\{(u, v) \in W^{1, p}(\Omega) \times W^{1, q}(\Omega): \mathcal{F}(u, v) \leq c\right\}$.

Proof. Let $E_{n} \subset W^{1, p}(\Omega) \times W^{1, q}(\Omega)$ be a $n$-dimensional subspace such that $u,\left.v\right|_{\partial \Omega} \not \equiv 0$ for all $(u, v) \in E_{n}, u, v \neq 0$.

Hence, using $\left(\mathrm{F}_{8}\right)$ we have, for $(u, v) \in E_{n},\|u\|_{W^{1, p}(\Omega)}=1,\|v\|_{W^{1, q}(\Omega)}=1$,

$$
\begin{aligned}
\mathcal{F}\left(t^{1 / p} u, t^{1 / q} v\right) & =\frac{t}{p}+\frac{t}{q}-\int_{\partial \Omega} F\left(x, t^{1 / p} u, t^{1 / q} v\right) \\
& \leq t\left(\frac{1}{p}+\frac{1}{q}\right)-t^{\theta} \int_{\partial \Omega} K(x, u, v) \\
& \leq t\left(\frac{1}{p}+\frac{1}{q}\right)-A_{n} t^{\theta}
\end{aligned}
$$

where

$$
A_{n}=\inf \left\{\int_{\partial \Omega} K(x, u, v):(u, v) \in E_{n},\|u\|_{W^{1, p}(\Omega)}=1,\|v\|_{W^{1, q}(\Omega)}=1\right\} .
$$

Observe that $A_{n}>0$ because $E_{n}$ is finite dimensional. As $\theta<1$ we obtain from (4.1) that there exists positive constants $\rho$ and $\varepsilon$ such that

$$
\mathcal{F}(u, v)<-\varepsilon \quad \text { for }(u, v) \in E_{n},\|u\|_{W^{1, p}(\Omega)}=\|v\|_{W^{1, q}(\Omega)}=\rho .
$$


Therefore, if we set $S_{\rho, n}=\left\{(u, v) \in E_{n}:\|u\|_{W^{1, p}(\Omega)}=\|v\|_{W^{1, q}(\Omega)}=\rho\right\}$, we have that $S_{\rho, n} \subset \mathcal{F}^{-\varepsilon}$. Hence by the monotonicity of the genus

$$
\gamma\left(\mathcal{F}^{-\varepsilon}\right) \geq \gamma\left(S_{\rho, n}\right)=n,
$$

as we wanted to show.

Finally, the following two Theorems end the proof of Theorem 1.2.

Theorem 4.1 Let

$$
\begin{aligned}
\Sigma= & \left\{A \subset W^{1, p}(\Omega) \times W^{1, q}(\Omega): A \text { is closed, } A=-A,\right. \\
& \text { and } \left.u,\left.v\right|_{\partial \Omega} \neq 0, \forall(u, v) \in A\right\}, \\
\Sigma_{k}=\{A \in \Sigma: \gamma(A) \geq k\}, &
\end{aligned}
$$

where $\gamma$ stands for the genus. Then

$$
c_{k}=\inf _{A \in \Sigma_{k}} \sup _{(u, v) \in A} \mathcal{F}(u, v)
$$

is a negative critical value of $\mathcal{F}$ and moreover, if $c=c_{k}=\cdots=c_{k+r}$, then $\gamma\left(K_{c}\right) \geq r+1$, where $K_{c}=\left\{(u, v) \in W^{1, p}(\Omega) \times W^{1, q}(\Omega): \mathcal{F}(u, v)=\right.$ $\left.c, \mathcal{F}^{\prime}(u, v)=0\right\}$.

Proof. The proof follows closely the one from Theorem 3.1 of [16]. According to Lemma 4.2 for every $k \in \mathbb{N}$ there exists $\varepsilon>0$ such that $\gamma\left(\mathcal{F}^{-\varepsilon}\right) \geq k$. As $\mathcal{F}$ is even and continuous it follows that $\mathcal{F}^{-\varepsilon} \in \Sigma_{k}$ therefore $c_{k} \leq-\varepsilon<0$. Moreover by Lemma 4.1, $\mathcal{F}$ is bounded below so $c_{k}>-\infty$. Let us now see that $c_{k}$ is in fact a critical value for $\mathcal{F}$. To this end let us suppose that $c=c_{k}=\cdots=c_{k+r}$. As $\mathcal{F}$ is even it follows that $K_{c}$ is symmetric. The Palais-Smale condition implies that $K_{c}$ is compact, therefore if $\gamma\left(K_{c}\right) \leq r$ by the continuity property of the genus (see [21]) there exists a neighborhood of $K_{c}, N_{\delta}\left(K_{c}\right)=\left\{(u, v) \in W^{1, p}(\Omega) \times W^{1, q}(\Omega)\right.$ : $\left.d\left((u, v), K_{c}\right) \leq \delta\right\}$, such that $\gamma\left(N_{\delta}\left(K_{c}\right)\right)=\gamma\left(K_{c}\right) \leq r$.

Now if $c_{k}$ is not a critical value of $\mathcal{F}$, using a standard deformation argument we arrive to a contradiction. See [16] for the details.

We end the section showing that the critical points of $\mathcal{F}$ form a compact set of $W^{1, p}(\Omega) \times W^{1, q}(\Omega)$.

Theorem 4.2 The set $K=\left\{(u, v) \in W^{1, p}(\Omega) \times W^{1, q}(\Omega): \mathcal{F}^{\prime}(u, v)=0\right\}$ is compact in $W^{1, p}(\Omega) \times W^{1, q}(\Omega)$.

Proof. As $\mathcal{F}$ is $C^{1}$ it is immediate that $K$ is closed. Let $\left(u_{j}, v_{j}\right)$ be a sequence in $K$. We have that, by $\left(\mathrm{F}_{3}\right)$ and the Sobolev trace theorem,

$$
\begin{aligned}
0 & =F^{\prime}\left(u_{j}, v_{j}\right)\left(u_{j}, v_{j}\right) \\
& \geq\left\|u_{j}\right\|_{W^{1, p}(\Omega)}^{p}+\left\|v_{j}\right\|_{W^{1, q}(\Omega)}^{q}-C\left(1+\left\|u_{j}\right\|_{W^{1, p}(\Omega)}^{r}+\left\|v_{j}\right\|_{W^{1, q}(\Omega)}^{s}\right) .
\end{aligned}
$$


As $1<r<p$ and $1<s<q$, we conclude that $\left(u_{j}, v_{j}\right)$ is bounded in $W^{1, p}(\Omega) \times$ $W^{1, q}(\Omega)$. Now we can use Palais-Smale condition to extract a convergent subsequence.

\section{$5 \quad$ Resonant case. $r=p$ and $s=q$}

In this section we deal with the resonant case, i.e. we assume $r=p, s=q$ in $\left(\mathrm{F}_{3}\right)$. First we need to study the eigenvalue problem (1.4). This problem is related to the one introduced in [2]. Our proofs follows closely the ones in [2], but since in our case the eigenvalue appears on the boundary condition, we include the details.

Lemma 5.1 Given $a \in L^{\infty}(\partial \Omega)$, there exists $\lambda_{1}(a) \in \mathbb{R}$ and $(\phi, \psi) \in W^{1, p}(\Omega) \times$ $W^{1, q}(\Omega)$ such that $(\phi, \psi)$ is a nontrivial solution of (1.4) with $\lambda=\lambda_{1}(a)$. Moreover

$\frac{1}{p}\|u\|_{W^{1, p}(\Omega)}^{p}+\frac{1}{q}\|v\|_{W^{1, q}(\Omega)}^{q}-\int_{\partial \Omega} a(x) G(u, v) d \sigma \geq \lambda_{1}(a)\left[\frac{1}{p}\|u\|_{L^{p}(\partial \Omega)}^{p}+\frac{1}{q}\|v\|_{L^{q}(\partial \Omega)}^{q}\right]$ for all $(u, v) \in W^{1, p}(\Omega) \times W^{1, q}(\Omega)$.

Proof. Choose $M>k\|a\|_{L^{\infty}(\partial \Omega)}$ where $k$ is the constant in $\left(\mathrm{G}_{2}\right)$. Then the functional

$$
\begin{aligned}
\mathcal{G}(u, v)= & \frac{1}{p}\|u\|_{W^{1, p}(\Omega)}^{p}+\frac{1}{q}\|v\|_{W^{1, q}(\Omega)}^{q}-\int_{\partial \Omega} a(x) G(u, v) d \sigma \\
& +M\left[\frac{1}{p}\|u\|_{L^{p}(\partial \Omega)}^{p}+\frac{1}{q}\|v\|_{L^{q}(\partial \Omega)}^{q}\right]
\end{aligned}
$$

is nonnegative for $(u, v) \in W^{1, p}(\Omega) \times W^{1, q}(\Omega)$. Let

$$
S=\left\{(u, v) \in W^{1, p}(\Omega) \times W^{1, q}(\Omega): \frac{1}{p}\|u\|_{L^{p}(\partial \Omega)}^{p}+\frac{1}{q}\|v\|_{L^{q}(\partial \Omega)}^{q}=1\right\}
$$

Let $\mu=\inf \{\mathcal{G}(u, v):(u, v) \in S\}$. Let us take $\left(u_{n}, v_{n}\right) \in S$ a minimizing sequence for $\mathcal{G}$. It follows that $\left(u_{n}, v_{n}\right)$ is bounded in $W^{1, p}(\Omega) \times W^{1, q}(\Omega)$. Then, by taking a subsequence if necessary, $\left(u_{n}, v_{n}\right)$ converges weakly in $W^{1, p}(\Omega) \times W^{1, q}(\Omega)$ and strongly in $L^{p}(\partial \Omega) \times L^{q}(\partial \Omega)$ to some $(\phi, \psi)$.

Passing to the limit we obtain $\mathcal{G}(\phi, \psi) \leq \mu$ which is in fact an equality since $(\phi, \psi) \in S$. So the infimum is achieved. It follows then that $(\phi, \psi)$ verifies

$$
\begin{cases}\Delta_{p} \phi=|\phi|^{p-2} \phi, & \text { in } \Omega, \\ |\nabla \phi|^{p-2} \frac{\partial \phi}{\partial \nu}-a(x) G_{u}(\phi, \psi)+M|\phi|^{p-2} \phi=\mu_{M}|\phi|^{p-2} \phi, & \text { on } \partial \Omega \\ \Delta_{q} \psi=|\psi|^{q-2} \psi, & \text { in } \Omega \\ |\nabla \psi|^{q-2} \frac{\partial \psi}{\partial \nu}-a(x) G_{v}(\phi, \psi)+M|\psi|^{q-2} \psi=\mu_{M}|\psi|^{q-2} \psi, & \text { on } \partial \Omega\end{cases}
$$


where $\mu_{M}$ is the Lagrange multiplier. It is easy to check that $\left(\mathrm{G}_{1}\right)$ implies

$$
G(u, v)=\frac{1}{p} u G_{u}(u, v)+\frac{1}{q} v G_{v}(u, v) .
$$

From the minimization problem, using (5.2), it is now easy to see that $\mu=\mu_{M}$ so the lemma follows by taking $\lambda_{1}(a)=\mu-M$.

Remark 5.1 From the minimization argument both $\phi$ and $\psi$ can be taken to be nonnegative. Also, by known regularity results (see for example [23]) the eigenfuntions $(\phi, \psi)$ are $C^{1}(\bar{\Omega})$. Then, by the maximum principle and Hopf's Lemma (see [24]), we can assume that $\phi$ and $\psi$ are either positive functions or vanishes identically in $\bar{\Omega}$. In either case, one of $\phi$ or $\psi$ is strictly positive.

Lemma $5.2 \lambda_{1}(a)$ is continuous with respect to a in the $L^{\infty}{ }_{-n o r m}$.

Proof. Let $a, b \in L^{\infty}(\partial \Omega)$ and let $\mathcal{G}_{a}$ and $\mathcal{G}_{b}$ be the associated functionals defined in (5.1). Given $\varepsilon>0$, choose $\left(u_{\varepsilon}, v_{\varepsilon}\right) \in S$ such that

$$
\mathcal{G}_{a}\left(u_{\varepsilon}, v_{\varepsilon}\right) \leq \lambda_{1}(a)+M+\frac{\varepsilon}{2} .
$$

Next, using $\left(\mathrm{G}_{2}\right)$ we obtain

$$
\left|\mathcal{G}_{b}\left(u_{\varepsilon}, v_{\varepsilon}\right)-\mathcal{G}_{a}\left(u_{\varepsilon}, v_{\varepsilon}\right)\right| \leq\|b-a\|_{L^{\infty}(\partial \Omega)} K
$$

where $K \geq \max \{k p, k q\}$. Now from (5.3) and (5.4) we obtain

$$
\begin{aligned}
\lambda_{1}(b)+M & \leq \mathcal{G}_{b}\left(u_{\varepsilon}, v_{\varepsilon}\right) \\
& \leq \mathcal{G}_{a}\left(u_{\varepsilon}, v_{\varepsilon}\right)+K\|b-a\|_{L^{\infty}(\partial \Omega)} \leq \lambda_{1}(a)+M+\frac{\varepsilon}{2}+K\|b-a\|_{L^{\infty}(\partial \Omega)}
\end{aligned}
$$

from where the continuity follows.

We say that the functional $\mathcal{F}$ satisfies the Cerami condition if for every sequence $\left(u_{n}, v_{n}\right) \in W^{1, p}(\Omega) \times W^{1, q}(\Omega)$ such that

$$
\left|\mathcal{F}\left(u_{n}, v_{n}\right)\right| \leq C, \quad\left(1+\left\|u_{n}\right\|_{W^{1, p}(\Omega)}+\left\|v_{n}\right\|_{W^{1, q}(\Omega)}\right) \mathcal{F}^{\prime}\left(u_{n}, v_{n}\right) \rightarrow 0
$$

there exists a subsequence that converges strongly in $W^{1, p}(\Omega) \times W^{1, q}(\Omega)$.

Lemma 5.3 Under the hypotheses $\left(\mathrm{F}_{2}\right),\left(\mathrm{F}_{3}\right)$ and $\left(\mathrm{F}_{9}\right)$, the functional $\mathcal{F}$ satisfies the Cerami condition.

Proof. Let $\left(u_{n}, v_{n}\right)$ be a sequence that satisfies (5.5). Let us prove that $\left(u_{n}, v_{n}\right)$ is bounded in $W^{1, p}(\Omega) \times W^{1, q}(\Omega)$. 
From (5.5) it follows that

$$
\begin{aligned}
C & \geq-\mathcal{F}^{\prime}\left(u_{n}, v_{n}\right)\left(\frac{u_{n}}{p}, \frac{v_{n}}{q}\right)+\mathcal{F}\left(u_{n}, v_{n}\right) \\
& =\int_{\partial \Omega}\left(\frac{u_{n}}{p} F_{u}\left(x, u_{n}, v_{n}\right)+\frac{v_{n}}{q} F_{v}\left(x, u_{n}, v_{n}\right)-F(x, u, v)\right) d \sigma .
\end{aligned}
$$

Now, by $\left(\mathrm{F}_{9}\right)$ we get

$$
\int_{\partial \Omega}\left(\left|u_{n}\right|^{a}+\left|v_{n}\right|^{b}\right) d \sigma \leq C .
$$

As $a<p<p_{*}$ and $b<q<q_{*}$, by interpolation we get

$$
\begin{aligned}
\int_{\partial \Omega}\left|u_{n}\right|^{p} d \sigma & \leq\left(\int_{\partial \Omega}\left|u_{n}\right|^{a} d \sigma\right)^{\frac{p_{*}-p}{p_{*}-a}}\left(\int_{\partial \Omega}\left|u_{n}\right|^{p_{*}} d \sigma\right)^{\frac{p-a}{p_{*}-a}} \\
& \leq C\left(\int_{\partial \Omega}\left|u_{n}\right|^{p_{*}} d \sigma\right)^{\frac{p-a}{p_{*}-a}}
\end{aligned}
$$

Analogously, we get

$$
\int_{\partial \Omega}\left|v_{n}\right|^{q} d \sigma \leq C\left(\int_{\partial \Omega}\left|v_{n}\right|^{q_{*}} d \sigma\right)^{\frac{q-b}{q_{*}-b}}
$$

By the Sobolev trace inequality,

$$
\int_{\partial \Omega}\left|u_{n}\right|^{p} d \sigma \leq C\left\|u_{n}\right\|_{W^{1, p}(\Omega)}^{\frac{p_{*}(p-a)}{p_{*}-a}}, \quad \int_{\partial \Omega}\left|v_{n}\right|^{q} d \sigma \leq C\left\|v_{n}\right\|_{W^{1, q}(\Omega)}^{\frac{q_{*}(q-b)}{q_{*}-b}} .
$$

Finally, by $\left(\mathrm{F}_{3}\right)$ and (5.6), we have

$$
\mathcal{F}\left(u_{n}, v_{n}\right) \geq \frac{\left\|u_{n}\right\|_{W^{1, p}(\Omega)}^{p}}{p}+\frac{\left\|v_{n}\right\|_{W^{1, q}(\Omega)}^{q}}{q}-C\left(\left\|u_{n}\right\|_{W^{1, p}(\Omega)}^{\frac{p_{*}(p-a)}{p_{*}-a}}+\left\|v_{n}\right\|_{W^{1, q}(\Omega)}^{\frac{q_{*}(q-b)}{q_{*}-b}}\right) .
$$

As $\mathcal{F}\left(u_{n}, v_{n}\right)$ is bounded, $\frac{p_{*}(p-a)}{p_{*}-a}<p$ and $\frac{q_{*}(q-b)}{q_{*}-b}<q$ the boundedness of the sequence follows. Now, the proof of the Lemma follows as in the end of Lemma 3.2.

Finally we are ready to prove Theorem 1.3.

Proof of Theorem 1.3. It remains to show that $\mathcal{F}$ has the Mountain Pass Geometry, since the compactness is implied by Lemma 5.3 .

First we prove that $(0,0)$ is a local minimum. By the second line in $\left(\mathrm{F}_{10}\right)$ and $\left(\mathrm{F}_{3}\right)$ there exists a constant $C>0$ such that

$$
F(x, u, v) \leq b(x) \bar{G}(u, v)+C\left(|u|^{\tilde{r}}+|v|^{\tilde{s}}\right),
$$

for all $x \in \partial \Omega$ and $u, v \in \mathbb{R}$ where $\tilde{r}>p$ and $\tilde{s}>q$. 
For some $\delta>0$ small to be chosen, we have

$$
\begin{aligned}
\mathcal{F}(u, v) \geq & \delta\left(\frac{\|u\|_{W^{1, p}(\Omega)}^{p}}{p}+\frac{\|v\|_{W^{1, q}(\Omega)}^{q}}{q}\right) \\
& +(1-\delta)\left(\frac{\|u\|_{W^{1, p}(\Omega)}^{p}}{p}+\frac{\|v\|_{W^{1, q}(\Omega)}^{q}}{q}-\int_{\partial \Omega} \frac{b(x)}{1-\delta} \bar{G}(u, v) d \sigma\right) \\
& -C\|u\|_{W^{1, p}(\Omega)}^{\tilde{r}}-C\|v\|_{W^{1, q}(\Omega)}^{\tilde{s}} .
\end{aligned}
$$

By Lemma 5.2, for $\delta$ small enough

$$
\frac{\|u\|_{W^{1, p}(\Omega)}^{p}}{p}+\frac{\|v\|_{W^{1, q}(\Omega)}^{q}}{q}-\int_{\partial \Omega} \frac{b(x)}{1-\delta} \bar{G}(u, v) d \sigma>0 .
$$

Hence

$$
\mathcal{F}(u, v) \geq \delta\left(\frac{\|u\|_{W^{1, p}(\Omega)}^{p}}{p}+\frac{\|v\|_{W^{1, q}(\Omega)}^{q}}{q}\right)-C\|u\|_{W^{1, p}(\Omega)}^{\tilde{r}}-C\|v\|_{W^{1, q}(\Omega)}^{\tilde{s}} .
$$

So if $\|u\|_{W^{1, p}(\Omega)},\|v\|_{W^{1, q}(\Omega)}=\rho$ with $\rho$ small, then $\mathcal{F}(u, v)>\varepsilon>0$.

Finally we use the first line in $\left(\mathrm{F}_{10}\right)$ to obtain

$$
\mathcal{F}(u, v) \leq \frac{\|u\|_{W^{1, p}(\Omega)}^{p}}{p}+\frac{\|v\|_{W^{1, q}(\Omega)}^{q}}{q}-\int_{\partial \Omega} a(x) G(u, v) d \sigma+C .
$$

Now we take $(u, v)=\left(t^{1 / p} \phi, t^{1 / q} \psi\right)$ in (5.7) where $(\phi, \psi)$ is the eigenfunction of Lemma 5.1 associated to $\lambda_{1}(a)$ to obtain

$$
\begin{aligned}
\mathcal{F}(u, v) & \leq t \frac{\|\phi\|_{W^{1, p}(\Omega)}^{p}}{p}+t \frac{\|\psi\|_{W^{1, q}(\Omega)}^{q}}{q}-t \int_{\partial \Omega} a(x) G(\phi, \psi) d \sigma+C \\
& =t \lambda_{1}(a)\left(\frac{1}{p} \int_{\partial \Omega}|\phi|^{p} d \sigma+\frac{1}{q} \int_{\partial \Omega}|\psi|^{q} d \sigma\right)+C \rightarrow-\infty,
\end{aligned}
$$

as $t \rightarrow+\infty$. This finishes the proof.

\section{Critical nonlinearity with superlinear perturbation}

In this section we study the critical case with a perturbation. We consider $F_{\lambda}(x, u, v)=F^{c}(x, u, v)+\lambda F^{s}(x, u, v)$ where $F^{c}$ and $F^{s}$ satisfy the assumptions of Theorem 1.4.

To prove our existence result, since we have lost the compactness in the inclusions $W^{1, p}(\Omega) \hookrightarrow L^{p_{*}}(\partial \Omega)$ and $W^{1, q}(\Omega) \hookrightarrow L^{q_{*}}(\partial \Omega)$, we can no longer expect 
the Palais-Smale condition to hold. Anyway we can prove a local Palais-Smale condition that will hold for $\mathcal{F}(u, v)$ below a certain value of energy. The technical result used here, the concentrated compactness method, is mainly due to [18, 19].

Let $\left(u_{j}, v_{j}\right)$ be a bounded sequence in $W^{1, p}(\Omega) \times W^{1, q}(\Omega)$ then there exists a subsequence that we still denote $\left(u_{j}, v_{j}\right)$, such that

$$
\begin{array}{lclc}
u_{j} \rightarrow u & \text { weakly in } W^{1, p}(\Omega), & v_{j} \rightarrow v & \text { weakly in } W^{1, q}(\Omega), \\
u_{j} \rightarrow u & \text { strongly in } L^{r}(\partial \Omega), & v_{j} \rightarrow v & \text { strongly in } L^{s}(\partial \Omega), \\
\left|\nabla u_{j}\right|^{p} \rightarrow d \mu_{p}, & \left.\left|u_{j}\right| \partial \Omega\right|^{p_{*}} \rightarrow d \eta_{p}, & \left|\nabla v_{j}\right|^{q} \rightarrow d \mu_{q},\left.\quad\left|v_{j}\right| \partial \Omega\right|^{q_{*}} \rightarrow d \eta_{q},
\end{array}
$$

weakly-* in the sense of measures. We observe that $d \eta_{p}$ and $d \eta_{q}$ are measures supported on $\partial \Omega$.

Now we state the following Lemma due to $[18,19]$.

Lemma 6.1 Let $u_{j}$ be a weakly convergent sequence in $W^{1, p}(\Omega)$ with weak limit $u$ such that

$$
\left|\nabla u_{j}\right|^{p} \rightarrow d \mu \quad \text { and }\left.\quad\left|u_{j}\right|_{\partial \Omega}\right|^{p_{*}} \rightarrow d \eta
$$

weakly-* in the sense of measures. Then there exists $x_{1}, \ldots, x_{l} \in \partial \Omega$ such that

(1) $d \eta=|u|^{p_{*}}+\sum_{j=1}^{l} \eta_{j} \delta_{x_{j}}, \eta_{j}>0$,

(2) $d \mu \geq|\nabla u|^{p}+\sum_{j=1}^{l} \mu_{j} \delta_{x_{j}}, \mu_{j}>0$,

(3) $\left(\eta_{j}\right)^{\frac{p}{p_{*}}} \leq \frac{\mu_{j}}{S_{p}}$, where $S_{p}=S_{p}(\Omega)$ is the best constant in the Sobolev trace inequality $S_{p}\|u\|_{L^{p_{*}}(\partial \Omega)}^{p} \leq\|u\|_{W^{1, p}(\Omega)}^{p}$.

Next, we use Lemma 6.1 to prove a local Palais-Smale condition.

Lemma 6.2 Let $\left(u_{j}, v_{j}\right) \subset W^{1, p}(\Omega) \times W^{1, q}(\Omega)$ be a Palais-Smale sequence for $\mathcal{F}$, with energy level $c$. If

$$
c<C \min \left\{S_{p}^{\frac{p_{*}}{p_{*}-p}}, S_{q}^{\frac{q_{*}}{q_{*}-q}}\right\},
$$

where $C$ is a constant that depends only on the bounds for $F^{c}, p, q$ and $N$ but is otherwise independent of $\Omega$. Then there exists a subsequence $\left(u_{j_{k}}, v_{j_{k}}\right)$ that converges strongly in $W^{1, p}(\Omega) \times W^{1, q}(\Omega)$.

Proof. From the fact that $\left(u_{j}, v_{j}\right)$ is a Palais-Smale sequence it follows, by $\left(\mathrm{F}_{5}\right)$ and $\left(\mathrm{F}_{2}^{c}\right)$ that $\left(u_{j}, v_{j}\right)$ is bounded in $W^{1, p}(\Omega) \times W^{1, q}(\Omega)$ (see Lemma 3.2). By Lemma 6.1 there exists a subsequence, that we still denote $\left(u_{j}, v_{j}\right)$, such that

$$
\begin{aligned}
& \left(u_{j}, v_{j}\right) \rightarrow(u, v) \text { weakly in } W^{1, p}(\Omega) \times W^{1, q}(\Omega), \\
& \left(u_{j}, v_{j}\right) \rightarrow(u, v) \text { in } L^{r}(\partial \Omega) \times L^{s}(\partial \Omega), \text { and a.e. in } \partial \Omega, \\
& \left(u_{j}, v_{j}\right) \rightarrow(u, v) \text { in } L^{p}(\Omega) \times L^{q}(\Omega), \text { and a.e. in } \Omega,
\end{aligned}
$$




$$
\begin{aligned}
& \left|\nabla u_{j}\right|^{p} \rightarrow d \mu_{p} \geq|\nabla u|^{p}+\sum_{k=1}^{l} \mu_{p, k} \delta_{x_{k}}, \\
& \left|\nabla v_{j}\right|^{q} \rightarrow d \mu_{q} \geq|\nabla v|^{q}+\sum_{i=1}^{m} \mu_{q, i} \delta_{y_{i}}, \\
& \left.\left|u_{j}\right|_{\partial \Omega}\right|^{p_{*}} \rightarrow d \eta_{p}=\left.|u|_{\partial \Omega}\right|^{p_{*}}+\sum_{k=1}^{l} \eta_{p, k} \delta_{x_{k}}, \\
& \left.\left|v_{j}\right|_{\partial \Omega}\right|^{q_{*}} \rightarrow d \eta_{q}=\left.|v|_{\partial \Omega}\right|^{q_{*}}+\sum_{i=1}^{m} \eta_{q, i} \delta_{y_{i}} .
\end{aligned}
$$

Assume first that there exists $x_{k} \neq y_{i}$ for every $i$. Then let $\phi \in C^{\infty}\left(\mathbb{R}^{N}\right)$ such that

$$
\phi \equiv 1 \text { in } B\left(x_{k}, \varepsilon\right), \quad \phi \equiv 0 \text { in } B\left(x_{k}, 2 \varepsilon\right)^{c}, \quad|\nabla \phi| \leq \frac{2}{\varepsilon},
$$

where $x_{k}$ belongs to the support of $d \eta_{p}$.

Consider $\left\{\left(u_{j} \phi, v_{j} \phi\right)\right\}$. Obviously this sequence is bounded in $W^{1, p}(\Omega) \times$ $W^{1, q}(\Omega)$. As $\mathcal{F}^{\prime}\left(u_{j}, v_{j}\right) \rightarrow 0$ in $W^{1, p}(\Omega)^{\prime} \times W^{1, q}(\Omega)^{\prime}$, we obtain that

$$
\lim _{j \rightarrow \infty}\left\langle\mathcal{F}^{\prime}\left(u_{j}, v_{j}\right) ;\left(\theta_{p} \phi u_{j}, \theta_{q} \phi v_{j}\right)\right\rangle=0 .
$$

By (6.2)-(6.3) we obtain,

$$
\begin{aligned}
\lim _{j \rightarrow \infty} & \int_{\Omega}\left|\nabla u_{j}\right|^{p-2} \nabla u_{j} \nabla \phi u_{j}+\left|\nabla v_{j}\right|^{q-2} \nabla v_{j} \nabla \phi v_{j} d x \\
= & \lim _{j \rightarrow \infty} \int_{\partial \Omega}\left(F_{u}^{c}\left(x, u_{j}, v_{j}\right) u_{j}+F_{v}^{c}\left(x, u_{j}, v_{j}\right) v_{j}\right) \phi d \sigma \\
& +\lambda \int_{\partial \Omega}\left(F_{u}^{s}\left(x, u_{j}, v_{j}\right) u_{j}+F_{v}^{s}\left(x, u_{j}, v_{j}\right) v_{j}\right) \phi d \sigma \\
& -\int_{\Omega} \phi\left(d \mu_{p}+d \mu_{q}\right)-\int_{\Omega}\left(|u|^{p}+|v|^{q}\right) \phi d x
\end{aligned}
$$

Using $\left(\mathrm{F}_{1}^{s}\right)$ and (6.2)-(6.3) it follows that

$$
\begin{gathered}
\lim _{j \rightarrow \infty} \int_{\partial \Omega}\left(F_{u}^{s}\left(x, u_{j}, v_{j}\right) u_{j}+F_{v}^{s}\left(x, u_{j}, v_{j}\right) v_{j}\right) \phi d \sigma \\
=\int_{\partial \Omega}\left(F_{u}^{s}(x, u, v) u+F_{v}^{s}(x, u, v) v\right) \phi d \sigma .
\end{gathered}
$$

Also, by $\left(\mathrm{F}_{1}^{c}\right)$ and Lemma 6.1 we have

$$
\lim _{j \rightarrow \infty} \int_{\partial \Omega}\left(F_{u}^{c}\left(x, u_{j}, v_{j}\right) u_{j}+F_{v}^{c}\left(x, u_{j}, v_{j}\right) v_{j}\right) \phi d \sigma \leq C \int_{\partial \Omega} \phi\left(d \eta_{p}+d \eta_{q}\right)
$$


Now, by Hölder inequality and weak convergence, we obtain

$$
\begin{aligned}
0 & \leq\left.\lim _{j \rightarrow \infty}\left|\int_{\Omega}\right| \nabla u_{j}\right|^{p-2} \nabla u_{j} \nabla \phi u_{j} d x \mid \\
& \leq \lim _{j \rightarrow \infty}\left(\int_{\Omega}\left|\nabla u_{j}\right|^{p} d x\right)^{(p-1) / p}\left(\int_{\Omega}|\nabla \phi|^{p}\left|u_{j}\right|^{p} d x\right)^{1 / p} \\
& \leq C\left(\int_{B\left(x_{k}, 2 \varepsilon\right) \cap \Omega}|\nabla \phi|^{p}|u|^{p} d x\right)^{1 / p} \\
& \leq C\left(\int_{B\left(x_{k}, 2 \varepsilon\right) \cap \Omega}|\nabla \phi|^{N} d x\right)^{1 / N}\left(\int_{B\left(x_{k}, 2 \varepsilon\right) \cap \Omega}|u|^{p N /(N-p)} d x\right)^{(N-p) / p N} \\
& \leq C\left(\int_{B\left(x_{k}, 2 \varepsilon\right) \cap \Omega}|u|^{p N /(N-p)} d x\right)^{(N-p) / p N} \rightarrow 0 \text { as } \varepsilon \rightarrow 0
\end{aligned}
$$

and by an analogous argument

$$
0=\lim _{j \rightarrow \infty} \int_{\Omega}\left|\nabla v_{j}\right|^{q-2} \nabla v_{j} \nabla \phi v_{j} d x .
$$

Then

$$
\begin{aligned}
0 \leq \liminf _{\varepsilon \rightarrow 0}[ & C \int_{\partial \Omega} \phi\left(d \eta_{p}+d \eta_{q}\right)+\lambda \int_{\partial \Omega}\left(F_{u}^{s}(x, u, v) u+F_{v}^{s}(x, u, v) v\right) \phi d \sigma \\
& \left.-\int_{\Omega} \phi\left(d \mu_{p}+d \mu_{q}\right)-\int_{\Omega}\left(|u|^{p}+|v|^{q}\right) \phi d x\right] \leq C \eta_{p, k}-\mu_{p, k} .
\end{aligned}
$$

By Lemma 6.1 we have that $\left(\eta_{p, k}\right)^{\frac{p}{p_{*}}} S_{p} \leq \mu_{p, k}$, therefore by (6.4) we obtain

$$
\left(\eta_{p, k}\right)^{\frac{p}{p *}} S_{p} \leq C \eta_{p, k} .
$$

Then, either $\eta_{p, k}=0$ or

$$
\eta_{p, k} \geq C S_{p}^{\frac{p_{*}}{p_{*}-p}}
$$

If (6.5) does indeed occur then, from the fact that $\left(u_{j}, v_{j}\right)$ is a Palais-Smale sequence, we obtain by $\left(\mathrm{F}_{2}^{c}\right)$ and $\left(\mathrm{F}_{5}\right)$,

$$
\begin{aligned}
c= & \lim _{j \rightarrow \infty} \mathcal{F}\left(u_{j}, v_{j}\right)=\lim _{j \rightarrow \infty} \mathcal{F}\left(u_{j}, v_{j}\right)-\left\langle\mathcal{F}^{\prime}\left(u_{j}, v_{j}\right) ;\left(\theta_{p} u_{j}, \theta_{q} v_{j}\right)\right\rangle \\
\geq & \int_{\partial \Omega} c\left(|u|^{p_{*}}+|v|^{q_{*}}\right) d \sigma+C S_{p}^{\frac{p_{*}}{p_{*}-p}} \\
& +\lambda \int_{\partial \Omega}\left(\theta_{p} F_{u}^{s}(x, u, v) u+\theta_{q} F_{v}^{s}(x, u, v) v-F^{s}(x, u, v)\right) d \sigma \\
\geq & C S_{p}^{\frac{p_{*}}{p_{*}-p}} .
\end{aligned}
$$


Observe that the constant $C$ depends only on the bounds for $F^{c}, p$ and $N$.

In an analogous way, we can prove that if there exists $y_{i} \neq x_{k}$ for every $k$ then $c \geq C S_{q}^{\frac{q_{*}}{q_{*}-q}}$, where again $C$ depends only on the bounds for $F^{c}, q$ and $N$.

Also, if there exists $x_{k}=y_{i}$ it follows that

$$
c \geq C\left(S_{p}^{\frac{p_{*}}{p_{*}-p}}+S_{q}^{\frac{q_{*}}{q_{*}-q}}\right) .
$$

So, if $c<C \min \left\{S_{p}^{\frac{p_{*}}{p_{*}-p}}, S_{q}^{\frac{q_{*}}{q_{*}-q}}\right\}$, we have

$$
\int_{\partial \Omega}\left|u_{j}\right|^{p_{*}} d \sigma \rightarrow \int_{\partial \Omega}|u|^{p_{*}} d \sigma, \quad \int_{\partial \Omega}\left|v_{j}\right|^{q_{*}} d \sigma \rightarrow \int_{\partial \Omega}|v|^{q_{*}} d \sigma
$$

and therefore $\left(u_{j}, v_{j}\right) \rightarrow(u, v)$ in $L^{p_{*}}(\partial \Omega) \times L^{q_{*}}(\partial \Omega)$. Now the proof finishes as usual.

Now we are ready to proceed with the proof of Theorem 1.4.

Proof of Theorem 1.4 In view of the previous result, we seek for critical values below level $c$. For that purpose, we want to use the Mountain Pass Lemma. Hence we have to check the following conditions:

(1) There exist constants $R, r>0$ such that if

$$
\frac{1}{p}\|u\|_{W^{1, p}(\Omega)}^{p}+\frac{1}{q}\|v\|_{W^{1, q}(\Omega)}^{q}=R \quad \Rightarrow \quad \mathcal{F}(u, v)>r .
$$

(2) There exists $\left(u_{0}, v_{0}\right) \in W^{1, p}(\Omega) \times W^{1, q}(\Omega)$ such that

$$
\left\|u_{0}\right\|_{W^{1, p}(\Omega)}+\left\|v_{0}\right\|_{W^{1, q}(\Omega)}>R \quad \text { and } \quad \mathcal{F}\left(u_{0}, v_{0}\right)<r .
$$

Let us first check (1). By $\left(\mathrm{F}_{1}^{c}\right),\left(\mathrm{F}_{6}\right)$ and the Sobolev trace Theorem we have,

$$
\begin{aligned}
\mathcal{F}(u, v)= & \frac{1}{p}\|u\|_{W^{1, p}(\Omega)}^{p}+\frac{1}{q}\|v\|_{W^{1, q}(\Omega)}^{q}-\int_{\partial \Omega} F_{\lambda}(x, u, v) d \sigma \\
\geq & \frac{1}{p}\|u\|_{W^{1, p}(\Omega)}^{p}+\frac{1}{q}\|v\|_{W^{1, q}(\Omega)}^{q}-C \int_{\partial \Omega}\left(|u|^{p_{*}}+|v|^{q_{*}}\right) d \sigma \\
& -\lambda C \int_{\partial \Omega}\left(|u|^{\bar{r}}+|v|^{\bar{s}}\right) d \sigma \\
\geq & \frac{1}{p}\|u\|_{W^{1, p}(\Omega)}^{p}+\frac{1}{q}\|v\|_{W^{1, q}(\Omega)}^{q}-C\left(\|u\|_{W^{1, p}(\Omega)}^{p_{*}}+\|v\|_{W^{1, q}(\Omega)}^{q_{*}}\right) \\
& -\lambda C\left(\|u\|_{W^{1, p}(\Omega)}^{\bar{r}}+\|v\|_{W^{1, q}(\Omega)}^{\bar{s}}\right) .
\end{aligned}
$$


Let

$$
g_{1}(t)=\frac{1}{p} t^{p}-C t^{p_{*}}-\lambda C t^{\bar{r}}, \quad g_{2}(t)=\frac{1}{q} t^{q}-C t^{q_{*}}-\lambda C t^{\bar{s}} .
$$

It is easy to check that $g_{i}(R)>r, i=1,2$ for some $R, r>0$.

Now, (2) is immediate as for a fixed $(w, z) \in W^{1, p}(\Omega) \times W^{1, q}(\Omega)$ with $w,\left.z\right|_{\partial \Omega} \not \equiv 0$ we have

$$
\lim _{t \rightarrow \infty} \mathcal{F}\left(t^{1 / p} w, t^{1 / q} z\right)=-\infty
$$

Now the candidate for critical value according to the Mountain Pass Theorem is

$$
c=\inf _{\phi \in \mathcal{C}} \sup _{t \in[0,1]} \mathcal{F}(\phi(t)),
$$

where $\mathcal{C}=\left\{\phi:[0,1] \rightarrow W^{1, p}(\Omega) \times W^{1, q}(\Omega) ;\right.$ continuous and $\phi(0)=(0,0), \phi(1)=$ $\left.\left(u_{0}, v_{0}\right)\right\}$. The problem is to show that $c$ verifies $(6.1)$ in order to apply the local Palais-Smale condition.

We fix $(w, z) \in W^{1, p}(\Omega) \times W^{1, q}(\Omega)$ with $\|w\|_{L^{p_{*}}(\partial \Omega)}=1$ and $\|z\|_{L^{q_{*}}(\partial \Omega)}=$ 1 , and define $h(t)=\mathcal{F}\left(t^{\theta_{p}} w, t^{\theta_{q}} z\right)$. We want to study the maximum of $h$. As $\lim _{t \rightarrow \infty} h(t)=-\infty$ it follows that there exists a $t_{\lambda}>0$ such that $\sup _{t>0}$ $\mathcal{F}\left(t^{\theta_{p}} w, t^{\theta_{q}} z\right)=h\left(t_{\lambda}\right)$. Differentiating we obtain, using $\left(\mathrm{F}_{1}^{c}\right)$,

$$
\begin{gathered}
0=h^{\prime}\left(t_{\lambda}\right)=t_{\lambda}^{\theta_{p} p-1} \theta_{p}\|w\|_{W^{1, p}(\Omega)}^{p}+t_{\lambda}^{\theta_{q} q-1} \theta_{q}\|z\|_{W^{1, q}(\Omega)}^{q} \\
\quad-\int_{\partial \Omega} \nabla F\left(x, t_{\lambda}^{\theta_{p}} w, t_{\lambda}^{\theta_{q}} z\right) \cdot\left(t_{\lambda}^{\theta_{p}-1} \theta_{p} w, t_{\lambda}^{\theta_{q}-1} \theta_{q} z\right) d \sigma \\
\leq t_{\lambda}^{\theta_{p} p-1} \theta_{p}\|w\|_{W^{1, p}(\Omega)}^{p}+t_{\lambda}^{\theta_{q} q-1} \theta_{q}\|z\|_{W^{1, q}(\Omega)}^{q}-c\left(t_{\lambda}^{\theta_{p} p_{*}-1}+t_{\lambda}^{\theta_{q} q_{*}-1}\right),
\end{gathered}
$$

from where it follows that, assuming that $\theta_{p} p \geq \theta_{q} q$,

$$
t_{\lambda}^{\theta_{p}\left(p_{*}-p\right)} \leq t_{\lambda}^{\theta_{p}\left(p_{*}-p\right)}+t_{\lambda}^{\theta_{q} q_{*}-\theta_{p} p} \leq C\left(\|w\|_{W^{1, p}(\Omega)}^{p}+t_{\lambda}^{\theta_{q} q-\theta_{p} p}\|z\|_{W^{1, q}(\Omega)}^{q}\right) .
$$

Hence $t_{\lambda}$ is bounded. Then, from (6.6) and our hypotheses $\left(\mathrm{F}_{5}\right)$ on $F^{s},\left(\mathrm{~F}_{1}^{c}\right)$ on $F^{c}$ and (3.2), we get

$$
0 \leq C\left(t_{\lambda}^{\theta_{p} p-1}+t_{\lambda}^{\theta_{q} q-1}-t_{\lambda}^{\theta_{p} p_{*}-1}-t_{\lambda}^{\theta_{q} q_{*}-1}-\lambda t_{\lambda} \int_{\partial \Omega} K(x, w, z)\right)
$$

we obtain that

$$
\lim _{\lambda \rightarrow \infty} t_{\lambda}=0
$$

On the other hand, it is easy to check that if $\lambda>\bar{\lambda}$ it must be $\mathcal{F}\left(t_{\bar{\lambda}}^{\theta_{p}} w, t_{\bar{\lambda}}^{\theta_{q}} z\right) \geq$ $\mathcal{F}\left(t_{\lambda}^{\theta_{p}} w, t_{\lambda}^{\theta_{q}} z\right)$, so by (6.7) we get

$$
\lim _{\lambda \rightarrow \infty} \mathcal{F}\left(t_{\lambda}^{\theta_{p}} w, t_{\lambda}^{\theta_{q}} z\right)=0 .
$$


But this identity means that there exists a constant $\bar{\Lambda}>0$ such that if $\lambda \geq \bar{\Lambda}$, then

$$
\sup _{t \geq 0} \mathcal{F}\left(t^{\theta_{p}} w, t^{\theta_{q}} z\right)<C \min \left\{S_{p}^{\frac{p_{*}}{p_{*}-p}}, S_{q}^{\frac{q_{*}}{q_{*}-q}}\right\}
$$

and the proof is finished if we choose $\left(u_{0}, v_{0}\right)=\left(t_{0}^{\theta_{p}} w, t_{0}^{\theta_{q}} z\right)$ with $t_{0}$ large in order to have $\mathcal{F}\left(t_{0}^{\theta_{p}} w, t_{0}^{\theta_{q}} z\right)<0$.

\section{Critical nonlinearity with sublinear perturbation}

In this section we study the critical case with a sublinear perturbation. We consider $F_{\lambda}(x, u, v)=F^{c}(x, u, v)+\lambda F^{s}(x, u, v)$ with the assumptions of Theorem 1.5.

The existence result is much easier in this case, since direct minimization applies as for $\lambda>0$ the infimum of $\mathcal{F}$ is negative and, for $\lambda$ small enough the local Palais-Smale condition can be used. For the multiplicity result, we will use ideas from [16].

We begin, as in the previous section, by using Lemma 6.1 to prove a local Palais-Smale condition.

Lemma 7.1 Let $\left(u_{j}, v_{j}\right) \subset W^{1, p}(\Omega) \times W^{1, q}(\Omega)$ be a Palais-Smale sequence for $\mathcal{F}$, with energy level $c$. If $c<C \min \left\{S_{p}^{\frac{p_{*}}{p_{*}-p}}, S_{q}^{\frac{q_{*}-q}{q_{*}}}\right\}-K\left(\lambda^{\frac{p_{*}}{p_{*}-r}}+\lambda^{\frac{q_{*}}{q_{*}-s}}\right)$, where $C$ and $K$ are positive constants independent of $\lambda$, then there exists a subsequence $\left(u_{j_{k}}, v_{j_{k}}\right)$ that converges strongly in $W^{1, p}(\Omega) \times W^{1, q}(\Omega)$.

Proof. From the fact that $\left(u_{j}, v_{j}\right)$ is a Palais-Smale sequence it follows by $\left(\mathrm{F}_{7}\right)$ and $\left(\mathrm{F}_{2}^{c}\right)$ that $\left(u_{j}, v_{j}\right)$ is bounded in $W^{1, p}(\Omega) \times W^{1, q}(\Omega)$ (see Lemma 3.2 and Lemma 6.2).

Now the proof follows exactly as in Lemma 6.2 until we get to

$$
\begin{aligned}
c \geq & \int_{\partial \Omega} c\left(|u|^{p_{*}}+|v|^{q_{*}}\right) d \sigma+C S_{p}^{\frac{p_{*}}{p_{*}-p}} \\
& +\lambda \int_{\partial \Omega}\left(\theta_{p} F_{u}^{s}(x, u, v) u+\theta_{q} F_{v}^{s}(x, u, v) v-F^{s}(x, u, v)\right) d \sigma
\end{aligned}
$$

where $(u, v)$ is the weak limit of $\left(u_{j}, v_{j}\right)$ in $W^{1, p}(\Omega) \times W^{1, q}(\Omega)$.

Using our hypothesis $\left(\mathrm{F}_{7}\right)$ and applying Hölder inequality, we find

$$
c \geq C S_{p}^{\frac{p_{*}}{p_{*}-p}}+c\left(\|u\|_{L^{p_{*}}(\partial \Omega)}^{p_{*}}+\|v\|_{L^{q_{*}}(\partial \Omega)}^{q_{*}}\right)-C \lambda\left(\|u\|_{L^{p_{*}}(\partial \Omega)}^{r}+\|v\|_{L^{q_{*}}(\partial \Omega)}^{S}\right) .
$$

Now, let $f(x, y)=c_{1}\left(x^{p_{*}}+y^{q_{*}}\right)-\lambda c_{2}\left(x^{r}+y^{s}\right)$. This function reaches its absolute minimum at $\left(x_{0}, y_{0}\right)=\left(\left(\frac{\lambda c_{2} r}{p_{*} c_{1}}\right)^{\frac{1}{p_{*}-r}},\left(\frac{\lambda c_{2} s}{q_{*} c_{1}}\right)^{\frac{1}{q_{*}-s}}\right)$, that is

$$
f(x, y) \geq f\left(x_{0}, y_{0}\right) \geq-K\left(\lambda^{\frac{p_{*}}{p_{*}-r}}+\lambda^{\frac{q_{*}}{q_{*}-s}}\right),
$$

where $K$ is independent of $\lambda$. 
Hence $c \geq C S_{p}^{\frac{p_{*}}{p_{*}-p}}-K\left(\lambda^{\frac{p_{*}}{p_{*}-r}}+\lambda^{\frac{q_{*}}{q_{*}-s}}\right)$, which contradicts our hypothesis. Therefore

$$
\lim _{j \rightarrow \infty} \int_{\partial \Omega}\left|u_{j}\right|^{p_{*}} d \sigma=\int_{\partial \Omega}|u|^{p_{*}} d \sigma
$$

and the rest of the proof is as that of Lemma 6.2.

Observe that $\left(\mathrm{F}_{1}^{c}\right)$ implies that

$$
c\left(|u|^{p_{*}}+|v|^{q_{*}}\right) \leq F^{c}(x, u, v) \leq C\left(|u|^{p_{*}}+|v|^{q_{*}}\right) .
$$

We now observe, using the Sobolev trace Theorem, $(7.1)$ and $\left(\mathrm{F}_{3}\right)$, that

$$
\begin{aligned}
\mathcal{F}(u, v) \geq & \frac{1}{p}\|u\|_{W^{1, p}(\Omega)}^{p}+\frac{1}{q}\|v\|_{W^{1, q}(\Omega)}^{q}-c_{1}\left(\|u\|_{W^{1, p}(\Omega)}^{p_{*}}+\|v\|_{W^{1, q}(\Omega)}^{q_{*}}\right) \\
& -\lambda c_{2}\left(\|u\|_{W^{1, p}(\Omega)}^{r}+\|v\|_{W^{1, q}(\Omega)}^{s}+1\right) \\
= & j_{1}\left(\|u\|_{W^{1, p}(\Omega)}\right)+j_{2}\left(\|v\|_{W^{1, q}(\Omega)}\right)-C,
\end{aligned}
$$

where $j_{1}(x)=\frac{1}{p} x^{p}-c_{1} x^{p_{*}}-\lambda c_{2} x^{r}$ and $j_{2}(y)=\frac{1}{q} y^{q}-c_{1} y^{q_{*}}-\lambda c_{2} y^{s}$. As $j_{i}$ attains a local but not a global minimum ( $j_{i}$ is not bounded below), we have to perform some sort of truncation. To this end let $x_{0}, x_{1}$ be such that $m<x_{0}<M<x_{1}$ where $m$ is the local minimum of $j_{1}$ and $M$ is the local maximum and $j_{1}\left(x_{1}\right)>j_{1}(m)$. For these values $x_{0}$ and $x_{1}$ we can choose a smooth function $\tau_{1}(x)$ such that $\tau_{1}(x)=1$ if $x \leq x_{0}, \tau_{1}(x)=0$ if $x \geq x_{1}$ and $0 \leq \tau_{1}(x) \leq 1$. Finally, let $\varphi_{1}(u)=\tau_{1}\left(\|u\|_{W^{1, p}(\Omega)}\right)$. Analogously, we define $\tau_{2}$ and $\varphi_{2}$ to perform the truncation in $j_{2}$. We define the truncated functional as follows

$$
\begin{aligned}
\tilde{\mathcal{F}}(u, v)= & \frac{1}{p} \int_{\Omega}|\nabla u|^{p}+|u|^{p} d x+\frac{1}{q} \int_{\Omega}|\nabla v|^{q}+|v|^{q} d x \\
& -\int_{\partial \Omega} F^{c}(x, u, v) \varphi_{1}(u) \varphi_{2}(v) d \sigma-\lambda \int_{\partial \Omega} F^{s}(x, u, v) d \sigma .
\end{aligned}
$$

As above, $\tilde{\mathcal{F}}(u, v) \geq \tilde{j}_{1}\left(\|u\|_{W^{1, p}(\Omega)}\right)+\tilde{j}_{2}\left(\|v\|_{W^{1, q}(\Omega)}\right)-C$ where $\tilde{j}_{1}(x)=\frac{1}{p} x^{p}-$ $c_{1} x^{p_{*}} \tau_{1}(x)-\lambda c_{2} x^{r}$ and $\tilde{j}_{2}(y)=\frac{1}{q} y^{q}-c_{1} y^{q_{*}} \tau_{2}(y)-\lambda c_{2} y^{s}$. We observe that if $x \leq x_{0}$ and $y \leq y_{0}$ then $\tilde{j}_{1}(x)=j_{1}(x), \tilde{j}_{2}(y)=j_{2}(y)$ and if $x \geq x_{1}$ then $\tilde{j}_{1}(x)=$ $\frac{1}{p} x^{p}-\lambda c_{2} x^{r}$ and if $y \geq y_{1}$ then $\tilde{j}_{2}(y)=\frac{1}{q} y^{q}-\lambda c_{2} y^{s}$.

Now we state a Lemma that contains the main properties of $\tilde{\mathcal{F}}$.

Lemma 7.2 $\tilde{\mathcal{F}}$ is $C^{1}$, if $\tilde{\mathcal{F}}(u, v) \leq 0$ then $\|u\|_{W^{1, p}(\Omega)}<x_{0},\|v\|_{W^{1, q}(\Omega)}<y_{0}$ and $\mathcal{F}(w, z)=\tilde{\mathcal{F}}(w, z)$ for every $(w, z)$ close enough to $(u, v)$. Moreover there exists $\Lambda>0$ such that if $0<\lambda<\Lambda$ then $\tilde{\mathcal{F}}$ satisfies a local Palais-Smale condition for $c \leq 0$. 
Proof. We only have to check the local Palais-Smale condition. Observe that every Palais-Smale sequence for $\tilde{\mathcal{F}}$ with energy level $c \leq 0$ must be bounded, therefore by Lemma 7.1 if $\lambda$ verifies $0<C \min \left\{S_{p}^{\frac{p_{*}}{p_{*}-p}}, S_{q}^{\frac{q_{*}}{q_{*}-q}}\right\}-K\left(\lambda^{\frac{p_{*}}{p_{*}-r}}+\lambda^{\frac{q_{*}}{q_{*}-s}}\right)$ then there exists a convergent subsequence.

Proof of Theorem 1.5. Existence part. As the perturbation $F^{s}$ is sublinear, it is easy to see that, for any $\lambda, \inf \tilde{\mathcal{F}}(u, v)<0$. In fact, one can check that if $t$ is small enough (depending on $\lambda$ ) and if $u,\left.v\right|_{\partial \Omega} \not \equiv 0$ then $\tilde{\mathcal{F}}(t u, t v)<0$. Now, for $\lambda$ small, the Palais-Smale condition holds below level 0 , therefore, a minimizing sequence has a convergent subsequence and the existence of a nontrivial solution follows.

The following Lemma gives the final ingredients needed in the proof of the multiplicity result of Theorem 1.5 .

Lemma 7.3 For every $n \in \mathbb{N}$ there exists $\varepsilon>0$ such that $\gamma\left(\tilde{\mathcal{F}}^{-\varepsilon}\right) \geq n$, where $\tilde{\mathcal{F}}^{-\varepsilon}=\{(u, v), \tilde{\mathcal{F}}(u, v) \leq-\varepsilon\}$.

Proof. The proof is analogous to that of Lemma 4.2.

Finally, we are ready to finish the proof of the Theorem.

Proof of Theorem 1.5. Multiplicity part. The proof is analogous to that of Theorem 1.2, here we use Lemma 7.1 and Lemma 7.3 instead of Lemma 4.1 and Lemma 4.2 respectively to work with the functional $\tilde{\mathcal{F}}$ and Lemma 7.2 to conclude on $\mathcal{F}$.

\section{References}

[1] A. AMBrosetTI and P. H. RABINOWITZ, Dual variational methods in critical point theory and applications, J. Funct. Anal. 14(4) (1973), 349-381.

[2] L. BOCCARDO and D. G. DE FIGUEIREDO, Some remarks on a system of quasilinear elliptic equations, NoDEA Nonlinear Differential Equations Appl. 9(3) (2002), 309-323.

[3] Ph. ClÉmENT, D. G. DE FIGUEIREDO and E. MITIDIERI, Positive solutions of semilinear elliptic systems, Comm. Partial Differential Equations 17 (1992), 923-940.

[4] C. V. COFFMAN, A minimum-maximum principle for a class of nonlinear integral equations, J. Anal. Math. 22 (1969), 391-419.

[5] D. G. COSTA and C. A. MAGALHÃES, Variational elliptic problems which are non-quadratic at infinity, Nonlinear Anal. TMA 23 (1994), 1401-1412.

[6] D. G. COSTA and C. A. MAGALHÃES, A variational approach to noncooperative elliptic systems, Nonlinear Anal. TMA 25 (1995), 699-715. 
[7] D. G. DE FIGUEIREDO, Positive solutions of semilinear elliptic equations, Springer Lecture Notes in Mathematics 957 (1982), 34-87.

[8] D. G. DE FIGUEIREDO, Semilinear elliptic systems: a survey of superlinear problems, Resenhas 2(4) (1996), 373-391.

[9] D. G. DE FIGUEIREDO and P. L. FELMER. On superquadratic elliptic systems, Trans. Amer. Math. Soc. 343 (1994), 99-116.

[10] D. G. DE FIGUEIREDO and C. A. MAGALHÃES, On nonquadratic hamiltonian elliptic systems, Advances Differential Equations 1(5) (1996), 881-898.

[11] P. FELMER, R. F. MANÁSEVICH and F. DE THÉLIN, Existence and uniqueness of positive solutions for certain quasilinear elliptic systems, Comm. Partial Differential Equations 17 (1992), 2013-2029.

[12] J. FERNÁNDEZ BONDER, Multiple positive solutions for quasilinear elliptic problems with sign-changing nonlinearities, Abstr. Appl. Anal. (12) (2004), $1047-1055$.

[13] J. FERNÁNDEZ BONDER, J. P. PINASCO and J. D. ROSSI, Existence results for a Hamiltonian elliptic system with nonlinear boundary conditions, Electron. J. Differential Equations 40 (1999), 1-15.

[14] J. FERNÁNDEZ BONDER, J. P. PINASCO and J. D. ROSSI, Infinitely many solutions for an elliptic system with nonlinear boundary conditions, Proceedings of the USA-Chile Workshop on Nonlinear Analysis (Viña del Mar-Valparaiso, 2000), 141-154 (electronic), Electron. J. Differ. Equ. Conf. 6, Southwest Texas, State Univ., San Marcos, TX, 2001.

[15] J. FERNÁNDEZ BONDER and J. D. ROSSI, Existence for an elliptic system with nonlinear boundary conditions via fixed point methods, Advances Differential Equations 6(1) (2001), 1-20.

[16] J. FERNÁNDEZ BONDER and J. D. ROSSI, Existence results for the $p$-Laplacian with nonlinear boundary conditions, J. Math. Anal. Appl. 263(1) (2001), 195-223.

[17] M. A. KRASNOSELSKI, Topological methods in the theory of nonlinear integral equations, Macmillan, New York, 1964.

[18] P.-L. LIONS, The concentration-compactness principle in the calculus of variations. The limit case, part 1, Rev. Mat. Iberoamericana 1(1) (1985), 145-201.

[19] P.-L. LIONS, The concentration-compactness principle in the calculus of variations. The limit case, part 2, Rev. Mat. Iberoamericana 1(2) (1985), 45-121. 
[20] S. MARTÍNEZ and J. D. ROSSI, Isolation and simplicity for the first eigenvalue of the $p$-laplacian with a nonlinear boundary condition, Abstr. Appl. Anal. 7(5) (2002), 287-293.

[21] P. RABINOWITZ, Minimax methods in critical point theory with applications to differential equations, CBMS Regional Conf. Ser. in Math. no. 65, Amer. Math. Soc., Providence, R.I., 1986.

[22] M. SCHECHTER and W. ZOU, An infinite-dimensional linking theorem and applications, J. Differential Equations 201(2) (2004), 324-350.

[23] P. TOLKSDORF, Regularity for a more general class of quasilinear elliptic equations, J. Differential Equations 51 (1984), 126-150.

[24] J. L. VAZQUEZ, A strong maximum principle for some quasilinear elliptic equations, Appl. Math. Optim. (1984), 191-202.

Received 16 June 2005; accepted 4 April 2006

Published Online First 13 August 2007

To access this journal online:

http://www.birkhauser.ch 